\title{
Changes of a frailty index based on common blood and urine tests during a hospital stay on geriatric wards predict 6-month and I-year mortality in older people
}

This article was published in the following Dove Medical Press journal:

Clinical Interventions in Aging

Jakob Jäger ${ }^{1,2}$

Cornel Christian Sieber ${ }^{1,3}$

Karl-Günter Gaßmann ${ }^{1,2}$

Martin Ritt ${ }^{1,2}$

'Institute for Biomedicine of Ageing (IBA), Friedrich-Alexander-Universität Erlangen-Nürnberg (FAU), D-90408

Nürnberg, Germany; ${ }^{2}$ Department of Internal Medicine III (Medicine of Ageing), Geriatrics Center Erlangen, Malteser Hospital Erlangen, D-9I054 Erlangen, Germany; ${ }^{3}$ Department of General Internal Medicine and Geriatrics, Hospital of the Order of St John of God, D-93049 Regensburg, Germany
Correspondence: Martin Ritt Institute for Biomedicine of Ageing (IBA), Friedrich-Alexander-Universität ErlangenNürnberg (FAU), Kobergerstraße 60,

D-90408 Nürnberg, Germany

Tel +49091318223702

$\mathrm{Fax}+4909|3| 8223703$

Email martin.ritt@waldkrankenhaus.de
Background: We aimed to evaluate the abilities of a 21-item frailty index based on laboratory blood and urine tests (FI-Lab21) assessed at different points in time, ie, at admission to hospital (FI-Lab2 $1_{\text {admission }}$ ) and before discharge from hospital (FI-Lab21 discharge $)$, and the change of the FI-Lab21 during the hospital stay to predict 6-month and 1-year mortality in hospitalized geriatric patients.

Methods: Five hundred hospitalized geriatric patients aged $\geq 65$ years were included in this analysis. Follow-up data were acquired after a period of 6 months and 1 year.

Results: The FI-Lab2 $1_{\text {admission }}$ and FI-Lab2 $1_{\text {discharge }}$ scores were $0.33 \pm 0.15$ and $0.31 \pm 0.14$, respectively $(P<0.001)$. The FI-Lab21 $1_{\text {admission }}$ and FI-Lab21 $1_{\text {discharge }}$ both predicted 6-month and 1-year mortality (areas under the receiver operating characteristic curves: $0.72,0.72,0.77$, and 0.75 , respectively, all $P<0.001$ ). The predictive abilities for 6-month and 1-year mortality of the FI-Lab2 $1_{\text {admission }}$ were inferior compared with those of the FI-Lab2 $1_{\text {discharge }}$ (all $P<0.05$ ). Patients with a reduction in or stable FI-Lab21 score during the hospital stay revealed lower 6-month and 1-year mortality rates compared with the persons whose FI-Lab21 score increased during the hospital stay (all $P<0.05$ ). After adjustment for age, sex, and FI-Lab2 $1_{\text {admission }}$, each 1\% decrease in the FI-Lab21 during the hospital stay was associated with a decrease in 6-month and 1 -year mortality of $5.9 \%$ and $5.3 \%$ (both $P<0.001$ ), respectively.

Conclusion: The FI-Lab21 assessed at admission or discharge and the changes of the FI-Lab21 during the hospital stay emerged as interesting and feasible approaches to stratify mortality risk in hospitalized geriatric patients.

Keywords: geriatric wards, frailty, frailty index, cumulative deficit, older people

\section{Plain language summary}

We evaluated the prognostic significance of a 21-item frailty index based on laboratory blood and urine tests (FI-Lab21) assessed at different points in time, ie, at admission to hospital (FI-Lab21 $1_{\text {admission }}$ ) and before discharge from the hospital (FI-Lab2 $1_{\text {discharge }}$ ), and the changes of the FI-Lab during the hospital stay of 513 geriatric inpatients on 6-month and 1-year mortality. The FI-Lab21 admission and FI-Lab21 discharge predicted 6-month and 1-year mortality. The FI-Lab2 $1_{\text {discharge }}$ was more powerful in predicting 6-month and 1-year mortality compared with the FI-Lab2 $1_{\text {admission }}$. The mean FI-Lab21 score could be reduced during the hospital stay. Patients with a reduction or stable FI-Lab21 score during the hospital stay demonstrated lower mortality rates after 6 months and 1 year of follow-up in comparison with the individuals whose FI-Lab21 score increased during the hospital stay. Moreover, taking potential confounders into account, an association between the quantity of the change of the FI-Lab during the hospital stay 
and 6-month and 1-year mortality was found. Thus, the FI-Lab21 evaluated at admission and/or assessed before discharge and the change of the FI-Lab during the hospital stay emerged as interesting and feasible instruments to evaluate the mortality risk of older individuals hospitalized on geriatric wards.

\section{Introduction}

Frailty describes a syndrome or clinical state accompanied by loss of physiological reserves, causing an increased vulnerability of an individual. ${ }^{1}$ Thereby frail individuals respond sensitively to external or internal stressor events with an even sudden deterioration of their health status. ${ }^{2}$ Due to this fluid condition, frail older people are at higher risk for adverse health outcomes such as falls, hospitalization, institutionalization, and mortality among others than robust persons. ${ }^{1,3-8}$ Adverse medical conditions going beyond physiological aging processes such as sarcopenia, ${ }^{9,10}$ malnutrition, ${ }^{11,12}$ multimorbidity, ${ }^{13}$ cognitive degradation, ${ }^{14}$ mental illness, ${ }^{15}$ mobility impairment, ${ }^{16}$ and chronic disease ${ }^{17}$ were found to conduce to frailty. Of clinical interest, frailty was proposed to be sensitive to adequate interventions and at least in part reversible in some individuals. ${ }^{18}$ There is general agreement among the importance of screening and/or assessing older people in relation to frailty and the imperative for developing feasible measure methods in older people. ${ }^{19}$

Several approaches to screen and/or assess individuals in relation to frailty have been elaborated, discussed, and validated. $^{20,21}$ The most accepted, applied, and widely studied are probably the frailty phenotype ${ }^{22}$ and the frailty index (FI) approaches. ${ }^{23}$ A limitation of the frailty phenotype is that it is inappropriate to investigate fine grades of frailty. ${ }^{24}$ This feasibility is a strength of an FI, enhancing precision and reliability for predicting adverse outcomes. ${ }^{25,26}$ FI is calculated by counting the number of present deficits (eg, clinical signs, symptoms, diseases, measures of function among others) of an individual divided by the number of all evaluated potential deficits. ${ }^{27}$ A limitation of FI established in clinical practice is that evaluation of a long checklist of clinical parameters and diseases is time-consuming. ${ }^{28}$ To overcome this aforementioned limitation of FI, recently FIs operationalized on the basis of standard laboratory tests (eg, blood tests, urine tests, blood pressure, and/or heart rate) (FI-Lab) have been introduced and validated. ${ }^{28-32}$

Several studies in different clinical settings found that FI-Lab predicts mortality in older people. ${ }^{28,33}$ We had previously found that FI-Lab operationalized from common blood and urine tests based on data that were obtained before discharge of the patients from hospital predicted 6-month and 1-year mortality in hospitalized patients at geriatric wards. ${ }^{28}$ Recent studies now indicate that FI operationalized from the data obtained at admission to hospital is able to predict mortality. ${ }^{3,33,34}$ From a clinician's perspective, FI-Lab obtained from data at admission to hospital might be preferable to FI-Lab before discharge, thereby allowing the information incorporated in the FI-Lab to be used for risk stratification and care planning already at the beginning of the hospital stay. Moreover, it might be of interest whether changes in FI-Lab during the hospital stay of the hospitalized geriatric patients reveal prognostic significance for mortality. Of note, no study has so far evaluated whether changes in FI-Lab during a hospital stay reveal prognostic power for mortality.

Several other authors have so far evaluated the power of an FI-Lab on mortality in different settings. ${ }^{29,30,32,42}$ Howlett et $\mathrm{al}^{42}$ assayed a strong relationship with mortality operationalizing an FI based on 21 routine blood tests combined with systolic and diastolic blood measurement in 1,013 community-dwelling and long-term institutionalized people aged 65 years or older during a 6 -year follow-up evaluation. Rockwood et $\mathrm{al}^{32}$ detected a predictive value of a 23 -item FI-Lab in 595 long-term care residents aged $82.7 \pm 8$ years in Canada during a 6-year follow-up analysis. A previous study by Blodgett et $\mathrm{al}^{29}$ in 8,888 participants composing a nationally representative sample (mean age of $49.4 \pm 19$ years) demonstrated higher mortality rates in individuals for higher FI-Lab categories during a period of 10-year follow-up. Another study by Blodgett et $\mathrm{al}^{30}$ in a sample of 3,369 community-dwelling men aged $60 \pm 11$ years accomplished in eight European countries found that FI-Lab predicted mortality, institutionalization, frequency of doctor visits, high number of medications, falls, and poor self-reported health status during a mean follow-up period of 4.4 years. In contrast to aforementioned studies, we operationalized FILab21 twice, first at admission and second before discharge.

In the study presented here, we aimed to evaluate whether 1) an FI-Lab operationalized from data obtained at admission to hospital predicts 6-month and 1-year mortality; 2) an FI-Lab operationalized from data obtained at admission to hospital differs from an FI-Lab operationalized from data obtained before discharge from hospital in the ability to predict 6-month and 1-year mortality; and 3) changes in the FI-Lab score during the hospital stay reveal predictive information for 6-month and 1-year mortality in patients hospitalized on geriatric wards. 


\section{Methods}

\section{Study design and study population}

This investigation was a secondary analysis concluding two recent prospective, longitudinal studies and summarized 513 patients, hospitalized on the geriatric wards of the Malteser Hospital Waldkrankenhaus St Marien in Erlangen, Germany. Criterion for inclusion was a minimum age of 65 years or older. Exclusion criteria were the missing of a written informed consent by the participant or by legal guardian on behalf of the participant. The patients were examined and evaluated for a variety of clinical characteristics and tests (eg, age, sex, weight, height, body mass index, functional impairment, diseases, Mini-Mental Status Examination, Barthel Index, Geriatric Depression Scale, Timed "Up and Go" Test). Furthermore, venous blood sampling and the analysis of spot urine took place at admission, before discharge, and, if necessary, at various points in time in-between during the hospital stay of the patients. Follow-up data were collected 6 months and 1 year after the baseline examination. These data included information with respect to death due to any cause among other clinical endpoints. The follow-up investigation was acquired by telephone interviews with participants, legal guardians, relatives, and/or general practitioners. The study protocol followed the Declaration of Helsinki and Good Clinical Practice and was approved by the Ethics Committee of the University of Erlangen-Nuremberg. Written informed consent was obtained from the study participants or their legal guardian prior to inclusion into the study.

\section{FI based on 20 laboratory blood tests and one urinary test (FI-Lab2I)}

The FI-Lab21 was composited out of 20 routine blood parameters and one parameter of a urine sample. In order to take care of a well-balanced combination, focus was on respecting various organ, health, and functional systems. Therefore, the FI-Lab21 as operationalized in this study presented here contained thyroid, liver, renal, hematological, inflammatory, electrolyte, and coagulation parameters. To prevent any imbalance, the number of parameters related to the red blood cell system was reduced compared with the 23-item FI-Lab in our previous study (ie, by removing the parameters hematocrit and hemoglobin) ${ }^{28}$ Much importance was attached to select parameters, which are available in any routine laboratory analysis. As also done in our preceding study ${ }^{28}$ every laboratory parameter was coded binary either 0 or 1; 0 denoted values within normal range, 1 demonstrated pathological values (ie, (sub)clinical deficits). For calculating FI-Lab21, a ratio between summed number of laboratory parameters that were out of the normal range and total number of examined parameters was built for each participant. To allege an example, an individual with pathological laboratory values for potassium, C-reactive protein, and total protein in spot urine, with normal values for the 18 further examined laboratory parameters, would have a summed number of three deficits, which yielded divided by 21 (total number of potential deficits) an FI-Lab21 score of 0.143 . As explained in this example comprehensibly, the FI-Lab21 score ranked in magnitude from 0 to 1 for each participant. If one or more parameter was not surveyed, the total number by which divided FI-Lab21 was given, decreased by the count of unavailable parameters. To set an example for this particular case, a patient with a deficit in five variables, two missing values and 14 values within normal range would have an FI-Lab21 of 0.263 (5/(21-2)). FI-Lab21 was calculated only up to a minimum number of 17 parameters, which guarantees $>80 \%$ of given variables for each case.

The FI-Lab21 was assessed twice during the hospital stay. One time with blood and urine sample obtained at admission (FI-Lab21 admission) and second time with samples gained before discharge (FI-Lab2 $1_{\text {discharge }}$ ). Moreover, the change in the FI-Lab21 during the hospital stay ( $\Delta$ FI-Lab21) of the study participants was calculated as difference between both aforementioned FI-Lab21s (FI-Lab21 $1_{\text {admission }}-$ FI-Lab21 $1_{\text {discharge }}$ ). If $\triangle$ FI-Lab21 assumed positive values, FI-Lab21 decreased during hospital stay (ie, FI-Lab21 at admission > FI-Lab21 before discharge), a negative $\Delta$ FI-Lab21 signals an increment of FI-Lab21 $1_{\text {discharge }}$ compared with FI-Lab21 $1_{\text {admission }}$.

\section{Statistical analyses}

Statistical analyses were accomplished by using IBM SPSS Statistics software (IBM Corp. Released 2017, IBM SPSS Statistics for Windows, version 25.0, IBM Corp., Armonk, NY, USA). Kolmogorov-Smirnov test was applied for testing normal distribution of values. Unless otherwise stated, results are presented as percentages, mean $\pm \mathrm{SD}$, or median (interquartile range). Comparing clinical characteristics was performed using chi-squared test and Mann-Whitney $U$-test where appropriate. Comparison of the change of the FI-Lab21 during the hospital stay, ie, FI-Lab2 $1_{\text {admission }}$ FI-Lab2 $1_{\text {discharge }}$, was performed using Wilcoxon signed-rank test. Furthermore, receiver operating characteristic curves 
were used to estimate area under the receiver operating characteristic curve (AUC) for FI-Lab21 $1_{\text {admission }}$, FI-Lab21 $1_{\text {discharge, }}$ and various laboratory parameters considered solely in relation to 6-month and 1-year mortality. An AUC value of 0.5 indicates a model of random classification. AUC values $>0.9$ denotes "very good," $>0.8$ "good," $>0.7$ "useful" predictive ability. ${ }^{35}$ Comparisons among AUCs were performed using the method of Hanley and McNeil. ${ }^{36}$ Kaplan-Meier survival analyses were applied to estimate the increased odds of mortality for each category increase in FI-Lab21 score $(0,0.001-0.1,0.101-0.2,0.201-0.3$, $0.301-0.4,0.401-0.5$, and $>0.5)$ and $\Delta$ FI-Lab21 score $(\geq 0$ and $<0$ ). For this model, $P$-value was observed by Breslow test. To determine predictive accuracy of FI-Lab21 $1_{\text {admission }}$ and FI-Lab21 $1_{\text {discharge }}$ for survival, Cox proportional hazard models were applied. For this aforementioned analysis, FI-Lab21 scores were multiplied by 100 and rounded to integers, converting FI-Lab21 scores to percentage values for modeling. HRs for the FI-Lab21 scores were both analyzed separately and adjusted for age and sex to take possible confounders into consideration. The level of statistical significance was set a priori at $P<0.050$.

\section{Results \\ Clinical characteristics of the study cohort}

A flowchart describing the process of inclusion and follow-up of the study participants is given in Figure 1. Five hundred thirteen patients were included in the study (see Figure 1). The clinical characteristics of these 513 individuals are given in Table 1 . For 13 of these 513 participants, $<80 \%$ of the laboratory parameters that construct the FI-Lab21 were available (see Figure 1). These 13 patients were excluded from further analyses. The clinical characteristics of these 13 patients are given in Table 1 . From all, the remaining 500 participants with availability of $80 \%$ or more of the laboratory parameters that construct the FI-Lab21 followup data could be obtained after 6 months of follow-up (see Figure 1). For six individuals, follow-up data could not be collected after 1-year of follow-up (see Figure 1). The clinical characteristics of the 500 patients with $80 \%$ or more FI-Lab21 parameters and the six individuals that were lost to follow-up after 1 year are shown in Table 2. Among aforementioned 500 individuals, the male participants were taller, revealed a higher body weight, and more often suffered from cancer, diabetes, and coronary infarction compared with the female individuals (see Table 2). After 6 months of follow-up, 86 of the 500 participants $(17.2 \%)$ had died due to any cause. After 1 year of follow-up, 114 of the 494 remaining study participants $(23.1 \%)$ had died.

\section{Distribution of the FI-Lab2 I at admission to hospital and before discharge from hospital}

The FI-Lab21 did not show a normal distribution irrespective of whether it was assessed at admission to hospital or before discharge from hospital (both $P<0.001$ ). With the FI-Lab2 $1_{\text {admission }}$, mean score was $0.33 \pm 0.15$, the median 0.33 , minimum 0 , maximum 0.76 , the first, fifth, 95th, and 99th percentiles observed were $0.05,0.09,0.57$, and 0.71 ,

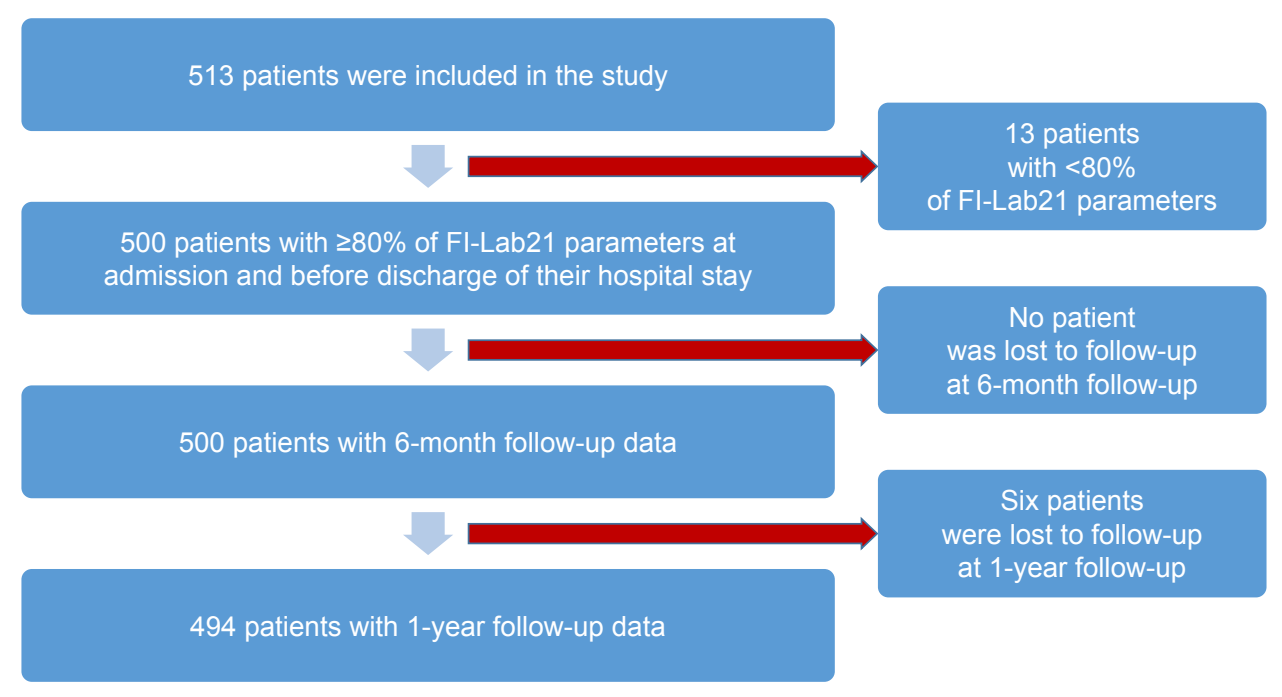

Figure I Flowchart. 
Table I Clinical characteristics at baseline examination for all study participants $(n=5$ I3) and participants with $<80 \%$ available $\mathrm{Fl}$-Lab2 I parameters $(n=13)$

\begin{tabular}{|c|c|c|}
\hline Clinical characteristics & $\begin{array}{l}\text { All participants } \\
(n=5 \mid 3)\end{array}$ & $\begin{array}{l}\text { Participants with }<80 \% \\
\text { FI-Lab2 I parameters }(n=13)\end{array}$ \\
\hline Female \% (n) & $67.4(346)$ & $84.6(\mathrm{II})$ \\
\hline Age (years) & $82.8 \pm 6.2$ & $83.2 \pm 5.4$ \\
\hline Height (cm) & $163 \pm 9.3$ & $160.8 \pm 5.9$ \\
\hline Weight (kg) & $72.2 \pm 16.2$ & $71.2 \pm 15.4$ \\
\hline BMI $\left(\mathrm{kg} / \mathrm{m}^{2}\right)$ & $27.1 \pm 5.5$ & $27.4 \pm 5.0$ \\
\hline Unintentional weight loss $>4.5 \mathrm{~kg}$ in last year \% (n) & $37(190)$ & $84.6(11)$ \\
\hline MMSE (points) & $25.5 \pm 4.3$ & $25.75 \pm 3.5$ \\
\hline GDS (points) & $4.1 \pm 2.7$ & $4.2 \pm 1.9$ \\
\hline Timed "up and go" test > 19 seconds or unable to perform \% (n) & $65.7(332)$ & $76.9(10)$ \\
\hline Barthel Index & $68.6 \pm 20.9$ & $63.5 \pm 23.9$ \\
\hline Heart failure \% (n) & $56.9(292)$ & $53.8(7)$ \\
\hline Peripheral vascular disease \% (n) & $14.8(76)$ & $0(0)$ \\
\hline Coronary infarction \% (n) & $12.5(64)$ & $7.7(1)$ \\
\hline Stroke \% (n) & $20.9(107)$ & I5.4 (2) \\
\hline Cancer \% (n) & I5.8 (8I) & $7.7(1)$ \\
\hline Diabetes mellitus \% ( $n$ ) & $37(190)$ & $61.5(8)$ \\
\hline Chronic lung disease \% (n) & $20.5(105)$ & I5.4 (2) \\
\hline Kidney disease \% (n) & $56.7(291)$ & $61.5(8)$ \\
\hline Urinary incontinence or catheterized \% ( $n$ ) & $38.8(199)$ & $61.5(8)$ \\
\hline Constipation \% (n) & $22.8(117)$ & $0(0)$ \\
\hline$>5$ medications $\%(n)$ & $96.3(494)$ & $92.3(12)$ \\
\hline
\end{tabular}

Note: Data are presented as mean \pm SD (unless otherwise stated).

Abbreviations: BMI, body mass index; FI-Lab2I, 2 I-item frailty index based on laboratory blood and urine tests; GDS, Geriatric Depression Scale; MMSE, Mini-Mental State Examination.

respectively. With the FI-Lab2 $1_{\text {discharge }}$, the mean score was $0.31 \pm 0.14$, median 0.32 , minimum measured 0 , maximum 0.71 , the first, fifth, 95th, and 99th percentiles ascertained were $0.00,0.10,0.53$, and 0.67 , respectively.

\section{Ability of the FI-Lab2 I at admission to hospital and before discharge from hospital to predict 6-month and I-year mortality}

The FI-Lab21 $1_{\text {admission }}$ and FI-Lab21 $1_{\text {discharge }}$ were both able to discriminate between individuals who had died and those who still were alive after 6 months and 1 year of follow-up (all $P<0.05$ ) (see Table 3). The ability of the FI-Lab21 to predict 6-month as well as 1-year mortality was greater at the point in time before discharge from hospital compared with the point in time at admission to hospital $(P=0.046$ and $P=0.040$, respectively). Among the individual laboratory parameters that construct the FI-Lab21, white blood cells, red blood cells, platelets, quick value, partial thromboplastin time, calcium, urea, total protein in serum, creatinine, aspartate aminotransferase, gamma-glutamyl transferase, lactate dehydrogenase, albumin, C-reactive protein, and total protein in spot urine were able to predict 6-month and/or 1-year mortality when assessed at admission to hospital or before discharge from hospital (all $P<0.05$ ) (see Table 3). With increasing scores of the FI-Lab21, the mortality rates of the study participants for 6 months and 1 year increased when the FI-Lab21 was applied at admission to hospital or before discharge from hospital (all $P<0.001$ ) (see Figures 2 and 3 and Table 4).

Each $1 \%$ increase in score of the FI-Lab2 $1_{\text {admission }}$ and FI-Lab21 $1_{\text {discharge }}$ was associated with an increase in mortality after 6 months and 1 year of follow-up ranging between $4.6 \%$ and $6.9 \%$, respectively (all $P<0.001$ ). After adjustment for the analyses of age and sex, each $1 \%$ increase in score of the FI-Lab2 $1_{\text {admission }}$ and FI-Lab2 $1_{\text {discharge }}$ was associated with an increase in mortality after 6 months and 1 year of follow-up of $4.9 \%$ and $7.1 \%$, respectively (all $P<0.001$ ) (see Table 5). 
Table 2 Clinical characteristics at baseline examination for all study participants with $>80 \%$ available FI-Lab2I parameters ( $\mathrm{n}=500$ ), divided by sex ( $n=165$ and $n=335$, respectively) and participants lost to follow-up at 1 -year follow-up after baseline examination ( $n=6$ )

\begin{tabular}{|c|c|c|c|c|c|}
\hline Clinical characteristics & $\begin{array}{l}\text { All participants } \\
\text { with }>\mathbf{8 0} \% \text { FI-Lab2 I } \\
\text { parameters }(n=500)\end{array}$ & $\begin{array}{l}\text { Male participants } \\
\text { with }>80 \% \text { FI-Lab2 I } \\
\text { parameters }(n=165)\end{array}$ & $\begin{array}{l}\text { Female participants } \\
\text { with }>80 \% \text { FI-Lab2 I } \\
\text { parameters } \\
(\mathrm{n}=335)\end{array}$ & $P$-value & $\begin{array}{l}\text { Participants lost } \\
\text { to follow-up at } \\
\text { I-year follow-up } \\
(n=6)\end{array}$ \\
\hline Female \% (n) & $67(335)$ & $0(0)$ & $100(335)$ & & $50(3)$ \\
\hline Age (years) & $82.8 \pm 6.2$ & $82.0 \pm 6.2$ & $83.2 \pm 6.2$ & 0.062 & $86 \pm 4.4$ \\
\hline Height $(\mathrm{cm})$ & $163.1 \pm 9.4$ & $171.8 \pm 7.7$ & $158.8 \pm 6.8$ & $<0.001$ & $166.2 \pm 8.6$ \\
\hline Weight (kg) & $72.2 \pm 16.2$ & $78.8 \pm 14.4$ & $68.9 \pm 16.1$ & $<0.001$ & $76.8 \pm 13.3$ \\
\hline BMI $\left(\mathrm{kg} / \mathrm{m}^{2}\right)$ & $27.1 \pm 5.6$ & $26.7 \pm 4.4$ & $27.3 \pm 6$ & 0.664 & $27.3 \pm 5.6$ \\
\hline $\begin{array}{l}\text { Unintentional weight loss }>4.5 \mathrm{~kg} \\
\text { in last year \% (n) }\end{array}$ & $36.2(I 8 I)$ & $41.8(69)$ & $33.4(I I 2)$ & 0.061 & $50(3)$ \\
\hline MMSE (points) & $25.5 \pm 4.3$ & $25.6 \pm 4.1$ & $25.5 \pm 4.4$ & 0.843 & $25 \pm 3.6$ \\
\hline GDS (points) & $4.1 \pm 2.7$ & $4.0 \pm 2.5$ & $4.2 \pm 2.8$ & 0.905 & $3.8 \pm 2.3$ \\
\hline $\begin{array}{l}\text { Timed "up and go" test }>19 \text { seconds } \\
\text { or unable to perform } \%(n)\end{array}$ & $64.4(322)$ & $65.5(108)$ & $63.9(2 \mid 4)$ & 0.730 & $66.7(4)$ \\
\hline Barthel Index & $68.7 \pm 20.8$ & $67.4 \pm 22.7$ & $69.4 \pm 19.8$ & 0.593 & $53.3 \pm 20.4$ \\
\hline Heart failure \% (n) & $42.8(2 \mid 4)$ & $45.5(75)$ & $4 I .5$ (I39) & 0.400 & $50(3)$ \\
\hline Peripheral vascular disease \% (n) & $15.2(76)$ & $19.4(32)$ & I3.1 (44) & 0.067 & $16.7(1)$ \\
\hline Coronary infarction \% (n) & $12.6(63)$ & $20.6(34)$ & $8.7(29)$ & $<0.001$ & $16.7(1)$ \\
\hline Stroke \% (n) & $21(105)$ & $23(38)$ & $20(67)$ & 0.434 & $16.7(1)$ \\
\hline Cancer \% (n) & $12(80)$ & $20.6(34)$ & $13.7(46)$ & 0.049 & $33.3(2)$ \\
\hline Diabetes mellitus \% ( $n$ ) & $36.4(182)$ & $43(7 \mathrm{I})$ & $33.1(111)$ & 0.031 & $0(0)$ \\
\hline Chronic lung disease \% (n) & $20.6(103)$ & $21.8(36)$ & $20(67)$ & 0.636 & $16.7(1)$ \\
\hline Kidney disease (\%) & $56.6(283)$ & $59.4(98)$ & $55.2(185)$ & 0.376 & $33.3(2)$ \\
\hline $\begin{array}{l}\text { Urinary incontinence or } \\
\text { catheterized \% (n) }\end{array}$ & $38.2(191)$ & $40(66)$ & $37.3(125)$ & $0.56 I$ & $66.7(4)$ \\
\hline Constipation \% (n) & $23.4(117)$ & $24.8(4 I)$ & $22.7(76)$ & 0.591 & $0(0)$ \\
\hline$>5$ medications $\%(n)$ & $96.4(482)$ & $97.6(161)$ & $95.8(32 \mathrm{I})$ & 0.322 & $100(6)$ \\
\hline
\end{tabular}

Note: Data are presented as mean \pm SD (unless otherwise stated).

Abbreviations: BMI, body mass index; FI-Lab2I, 21 -item frailty index based on laboratory blood and urine tests; GDS, Geriatric Depression Scale; MMSE, Mini-Mental State Examination.

\section{Ability of the change of the FI-Lab2 I between the point in time at admission to hospital and before discharge from hospital to predict 6-month and I-year mortality}

Among the individuals, the score of the FI-Lab21 decreased during the hospital stay with a mean reduction of the FI-Lab21 of $0.02 \pm 0.11(P<0.001)$, with a minimum of -0.48 and a maximum of 0.43 . The first, fifth, 95th, and 99th percentiles of the change in the FI-Lab21 during the hospital stay were $-0.25,-0.14,0.19$, and 0.29 , respectively (Figure 4 ). Individuals whose FI-Lab21 score decreased or did not change during the hospital stay had a lower risk to die during the 6-month and 1-year follow-up period compared with those individuals whose FI-Lab21 score increased during the hospital stay ( $P=0.004$ and $P=0.046$, respectively) (see Figure 5). Each 1\% decrease of FI-Lab21 during the hospital stay was associated with a decrease in 6-month mortality of $2.0 \%(P=0.040)$ and, with reference to statistical nonsignificant terms, a reduction in 1-year mortality of $1.3 \%$ ( $P=0.126)$ (see Table 6). After adjustment of the analysis for age, sex, and FI-Lab2 $1_{\text {admission}}$, each $1 \%$ decrease in the FI-Lab21 during the hospital stay was associated with a decrease in 6-month and 1-year mortality of 5.9\% and 5.3\% (both $P<0.001$ ), respectively (see Table 6).

\section{Discussion}

In this study, we show that FI-Lab21 operationalized from common blood and urine tests obtained at admission to hospital of hospitalized geriatric patients reveals predictive ability for 6-month and 1-year mortality. We thereby expand 


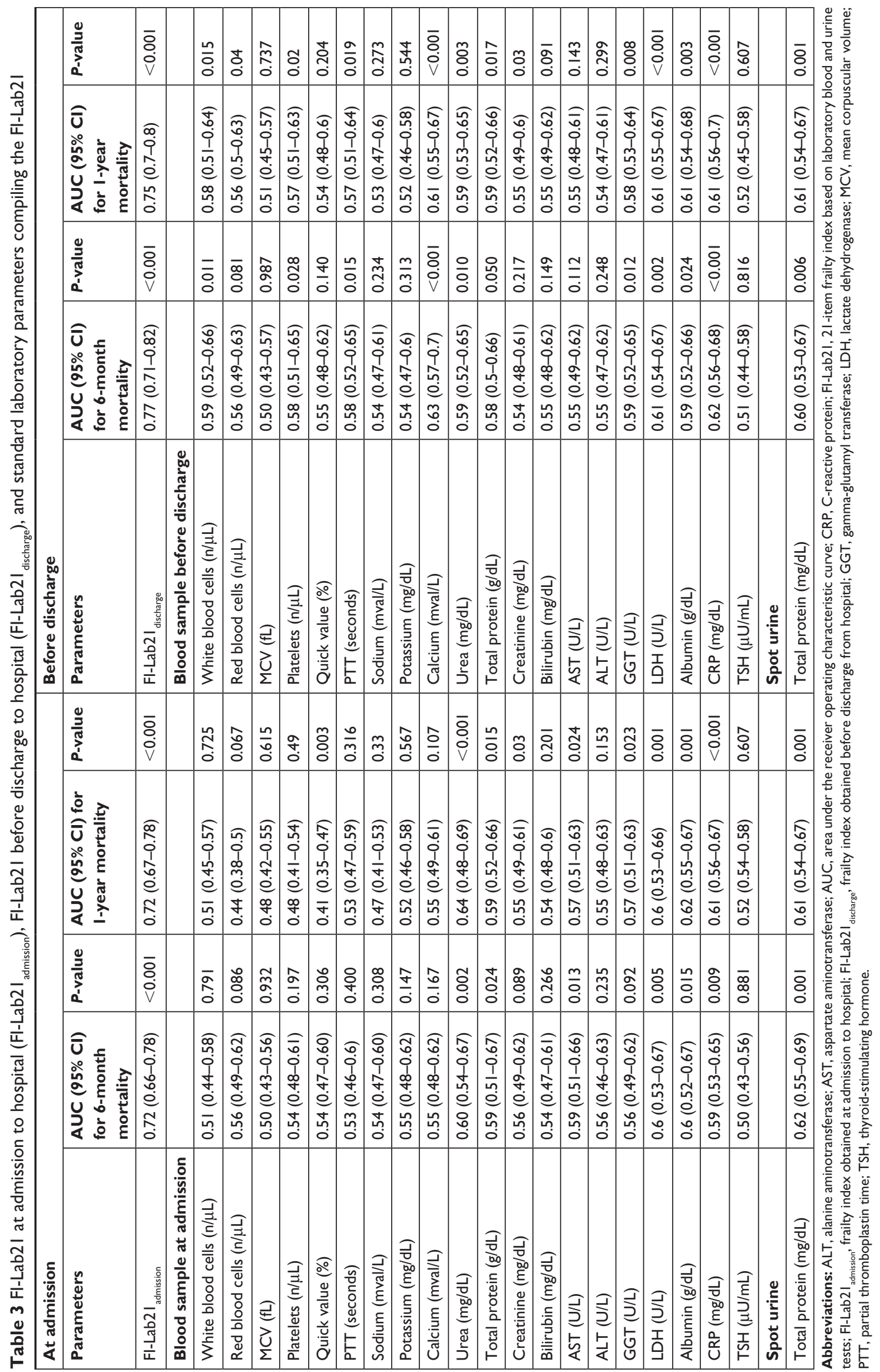




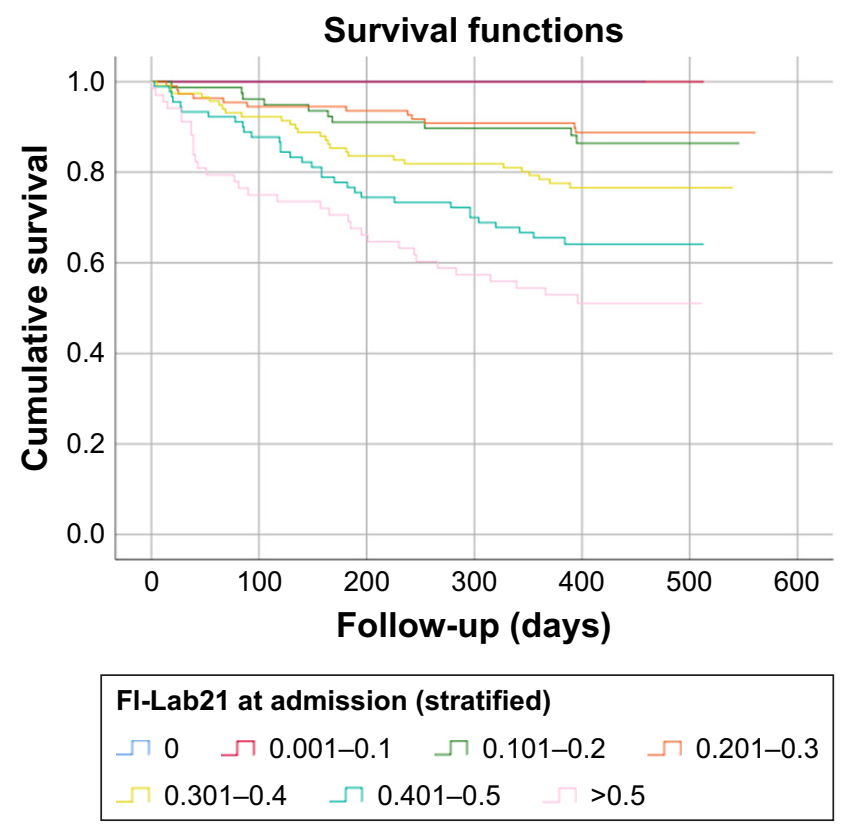

Figure 2 Kaplan-Meier survival function: Fl-Lab2 I at admission to hospital stratified into seven groups with increasing scores.

Abbreviation: FI-Lab2I, 21 -item frailty index based on laboratory blood and urine tests.

the findings of our previous study showing a predictive ability of FI-Lab based on blood and urine parameters obtained before discharge from hospital of hospitalized geriatric patients for 6-month and 1-year mortality. ${ }^{28}$ Our findings are in line with several other studies that demonstrated a

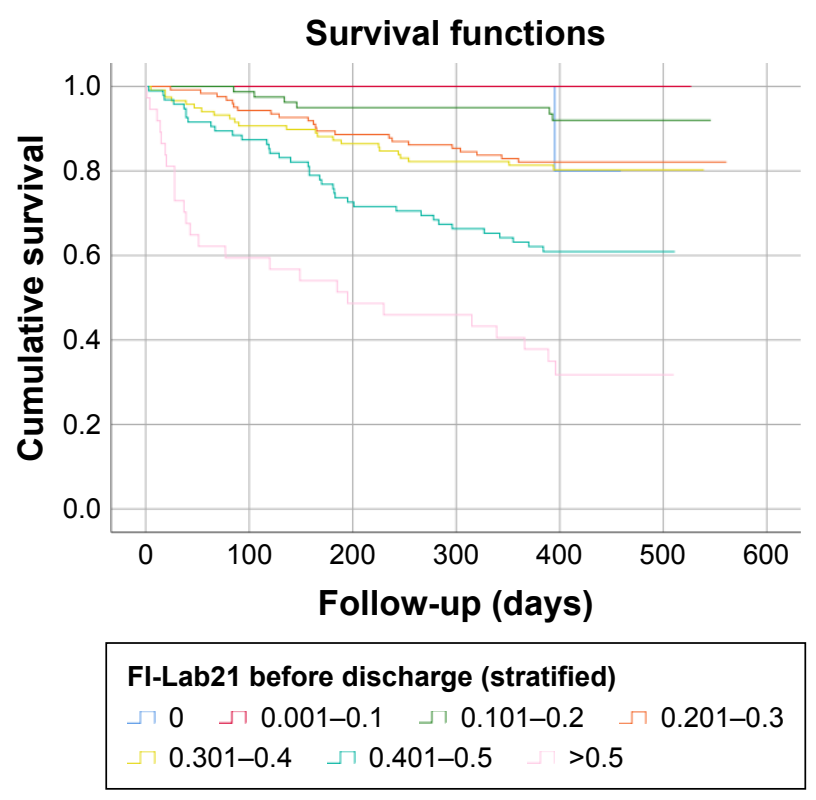

Figure 3 Kaplan-Meier survival function: Fl-Lab2I before discharge from hospital stratified into seven groups with increasing scores.

Abbreviation: FI-Lab2 I, $2 \mathrm{I}$-item frailty index based on laboratory blood and urine tests. predictive value of frailty instruments incorporating data that were obtained from patients at admission to the hospital for mortality or other adverse clinical outcomes. ${ }^{3,33,34}$ Klausen et $\mathrm{al}^{33}$ found a strong relation between FI-Lab based on 17 routine laboratory tests and 3-year mortality for 4,005 acutely admitted older patients aged 65 years or older at the Emergency Department of Copenhagen University Hospital, Denmark. Evans et $\mathrm{al}^{3}$ showed that FI based on a comprehensive geriatric assessment (CGA) that was administered at admission to general medical wards in a cohort of 752 hospitalized participants aged 75 years or older predicted institutionalization, length of hospital stay, and 30-day mortality. In a study by Hubbard et al in 1,418 Australian hospitalized patients aged 70 years or older, FI obtained within the first days of hospitalization on general medical units was predictive for length of hospital stay, discharge to residential aged care, falls, pressure ulcer incidence, and in-hospital mortality. ${ }^{34}$ This FI was operationalized based on a CGA including physical, mental, and cognitive functions, medications, medical diagnoses, and discharge destination..$^{34}$ In contrast to aforementioned studies, ${ }^{3,33,34}$ we obtained data for the FI-Lab21 not only at admission but also before discharge.

A further interesting finding of our study is that the FILab21 operationalized from blood and urine tests before discharge from hospital was more powerful compared with the FI-Lab21 obtained at admission to hospital. These findings indicate that the clinical state as evaluated with the FI-Lab21 before discharge from hospital after initial treatment of the acute disease or exacerbation of the chronic disease that had initially resulted in hospital admission stronger predicts 6-month and 1-year mortality than the more acute clinical state at hospital admission. The clinical state before discharge from hospital might probably better reflect to overall health status of a geriatric person than the clinical state driven by acute disease or exacerbation of the chronic disease at admission to the hospital geriatric ward of an older individual.

Of note, in our study during the hospital stay of the patients, the FI-Lab21 score decreased, ie, the FI-Lab21 operationalized from the data before discharge to hospital was lower compared with the FI-Lab21 based on data at admission to hospital. This indicates that the frailty severity of the patients, as assessed by the FI-Lab21, has decreased during their hospital stay. Possibly, the change in the FI-Lab21 between admission and discharge visualized performance and achievement of clinical interventions and therapies. Clearly, during their stay at the hospital geriatric wards, the patients received medical treatment and therapies by the physicians 
Table $4 \mathrm{FI}-\mathrm{Lab} 2 \mathrm{I}$ at admission to hospital $\left(\mathrm{FI}-\mathrm{Lab} 2 \mathrm{I}_{\text {admission }}\right)$, FI-Lab2 I before discharge from hospital $\left(\mathrm{FI}\right.$-Lab2 $\left.\mathrm{I}_{\text {discharge }}\right)$, and the change of the FI-Lab2I during the hospital stay $(\Delta \mathrm{FI}$-Lab2I) stratified according to score with 6-month and I-year mortality

\begin{tabular}{|c|c|c|c|c|c|}
\hline FI-Lab2 I score & $\begin{array}{l}\text { All patients, } \\
\%(n=494)\end{array}$ & $\begin{array}{l}\text { Six-month mortality, } \\
\%(n=86)\end{array}$ & $P$-value & $\begin{array}{l}\text { One-year mortality, } \\
\%(n=1 \mid 4)\end{array}$ & $P$-value \\
\hline FI-Lab2 I ${ }_{\text {admission }}$ & & & $<0.001$ & & $<0.001$ \\
\hline 0 & $0.0(n=2)$ & $0.0(n=0)$ & & $0.0(n=0)$ & \\
\hline $0.00 I-0.1$ & $6.3(n=31)$ & $0.0(n=0)$ & & $0.0(n=0)$ & \\
\hline $0.101-0.2$ & $15.8(n=78)$ & $10.3(n=8)$ & & $12.8(n=10)$ & \\
\hline $0.20 I-0.3$ & $22.1 \quad(n=109)$ & $6.4(n=7)$ & & $11.0(n=12)$ & \\
\hline $0.301-0.4$ & $23.5(n=116)$ & $16.8(n=20)$ & & $23.3(n=27)$ & \\
\hline $0.40 I-0.5$ & $18.2(n=90)$ & $26.1(n=24)$ & & $35.6(n=32)$ & \\
\hline$>0.5$ & $13.8(n=68)$ & $39.7(n=27)$ & & $48.5(n=33)$ & \\
\hline FI-Lab2 I discharge $_{\text {. }}$ & & & $<0.001$ & & $<0.001$ \\
\hline 0 & I.4 (n=7) & $0.0(n=0)$ & & $14.7(n=I)$ & \\
\hline $0.00 I-0.1$ & $6.9(n=34)$ & $0.0(n=0)$ & & $0.0(n=0)$ & \\
\hline $0.101-0.2$ & $16.2(n=80)$ & $4.9(n=4)$ & & $7.5(n=6)$ & \\
\hline $0.20 I-0.3$ & $24.9(n=123)$ & $11.4(n=14)$ & & $17.9(n=22)$ & \\
\hline $0.301-0.4$ & $23.9(n=118)$ & $15.6(n=19)$ & & $19.5(n=23)$ & \\
\hline $0.40 I-0.5$ & $19.2(n=95)$ & $30.2(n=29)$ & & $38.9(n=37)$ & \\
\hline$>0.5$ & $7.5(n=37)$ & $54 . I(n=20)$ & & $67.6(n=25)$ & \\
\hline$\Delta$ FI-Lab2 I & & & 0.004 & & 0.046 \\
\hline$\geq 0$ & $67.0(n=331)$ & $13.9(n=47)$ & & $20.8(n=69)$ & \\
\hline$<0$ & $33(n=163)$ & $23.9(n=39)$ & & $27.6(n=45)$ & \\
\hline
\end{tabular}

Note: $\Delta \mathrm{FI}$-Lab2 I = FI-Lab2 ${ }_{\text {admission }}-\mathrm{FI}$-Lab2 discharge .

Abbreviations: Fl-Lab2 I, $2 \mathrm{I}$-item frailty index based on laboratory blood and urine tests; $\mathrm{Fl}$-Lab2 $\mathrm{I}_{\text {admission }}$, frailty index obtained at admission to hospital; FI-Lab2 $\mathrm{I}_{\text {discharge' }}$ frailty index obtained before discharge from hospital.

and other members of the multidisciplinary geriatric team. Our findings are thus in line with the concept that frailty reveals a potentially reversible state at least in some patients and is sensitive to adequate treatment and therapy. ${ }^{37-41}$ In line with the findings of the study presented here, Basile et $\mathrm{al}^{37}$ revealed that a 46 -item FI based on laboratory and clinical data tended to decrease during the hospital stay in 156 hospitalized patients on geriatric wards being followed up to 12 months. Fairhall et $\mathrm{al}^{38}$ observed an increase in walking speed, more extended mobility, and a reduction of mobility-related disability in a randomized, controlled trail comprising 241 frail community-dwelling elderly in Sidney during a 12-month interventions program. Another randomized, controlled trial that was performed in 1994 by Fiatarone et al, ${ }^{39}$ showed an improvement of muscle size and strength as a result of a 10-week high-intensity resistance training of older frail people compared with nonexercising persons (mean age of the 100 nursing home residents was $87.1 \pm 0.6$ years). $\mathrm{Kim}^{40}$ investigated the effects of a 3-month exercise program and nutritional supplementation

Table $5 \mathrm{HR}$ and age- and sex-adjusted $\mathrm{HR}$ for each $0.0 \mathrm{I}$ increment in score of FI-Lab2 ${ }_{\text {admission }}$ and $\mathrm{FI}-$ Lab2 $\mathrm{I}_{\text {discharge }}$ in relation to 6-month and I-year mortality

\begin{tabular}{|c|c|c|c|c|}
\hline FI-Lab2 I ${ }_{\text {admission }}$, FI-Lab2 I discharge, and mortality & HR (95\% CI) & $P$-value & Adjusted HR (95\% Cl) & $P$-value \\
\hline \multicolumn{5}{|l|}{ Six-month mortality } \\
\hline FI-Lab2 I ${ }_{\text {admission }}($ per each $0.0 \mathrm{I}$ increment in score) & $1.046(1.032-1.061)$ & $<0.001$ & $1.049(1.033-1.064)$ & $<0.001$ \\
\hline 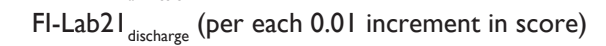 & $1.069(1.053-1.086)$ & $<0.001$ & I.07I (I.054-I.088) & $<0.001$ \\
\hline \multicolumn{5}{|l|}{ One-year mortality } \\
\hline FI-Lab2 I ${ }_{\text {admission }}($ per each 0.01 increment in score) & $1.046(1.034-1.059)$ & $<0.001$ & $1.048(1.035-1.061)$ & $<0.001$ \\
\hline FI-Lab2 $\mathrm{I}_{\text {discharge }}$ (per each 0.0I increment in score) & $1.065(1.05 \mathrm{I}-\mathrm{I} .08)$ & $<0.001$ & $1.066(1.05 \mathrm{I}-\mathrm{I} .08 \mathrm{I})$ & $<0.001$ \\
\hline
\end{tabular}

Abbreviations: Adjusted HR, hazard ratio adjusted for age and sex; Fl-Lab2I, 2 I-item frailty index based on laboratory blood and urine tests; Fl-Lab2 I admission, frailty index obtained at admission to hospital; FI-Lab2 $\mathrm{I}_{\text {discharge }}$, frailty index obtained before discharge from hospital. 


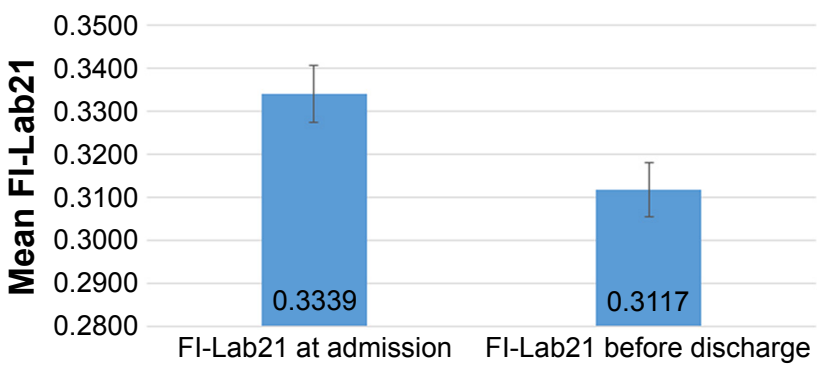

Figure 4 Mean change of the Fl-Lab2I during the hospital stay ( $n=500$; mean \pm standard error of the mean).

Abbreviation: $\mathrm{FI}-$ Lab2 I, $2 \mathrm{I}$-item frailty index based on laboratory blood and urine tests.

on improving frailty according to the frailty phenotype in 131 community-dwelling older people, finding an increase of muscle strength and bone mineral content. A recent randomized, controlled study performed in Singapore in 246 participants with mean age of $70 \pm 4.7$ years detected that nutritional, physical, and cognitive interventions were effective in reducing frailty (measured based on frailty phenotype criteria) during a follow-up period of 12 months. ${ }^{41}$ In contrast to our study, none of these authors operationalized an FI based solely on laboratory parameters. In line with the findings of this study all aforementioned studies showed changeability and improvement of frailty by purposive interventions. ${ }^{37-41}$

A further major finding of our study presented here is that the individuals who showed an increase of the FI-Lab21 during the hospital stay had a higher 6-month and 1-year mortality rate compared with those persons whose FI-Lab21 score did not change or decreased (ie, improved) during the

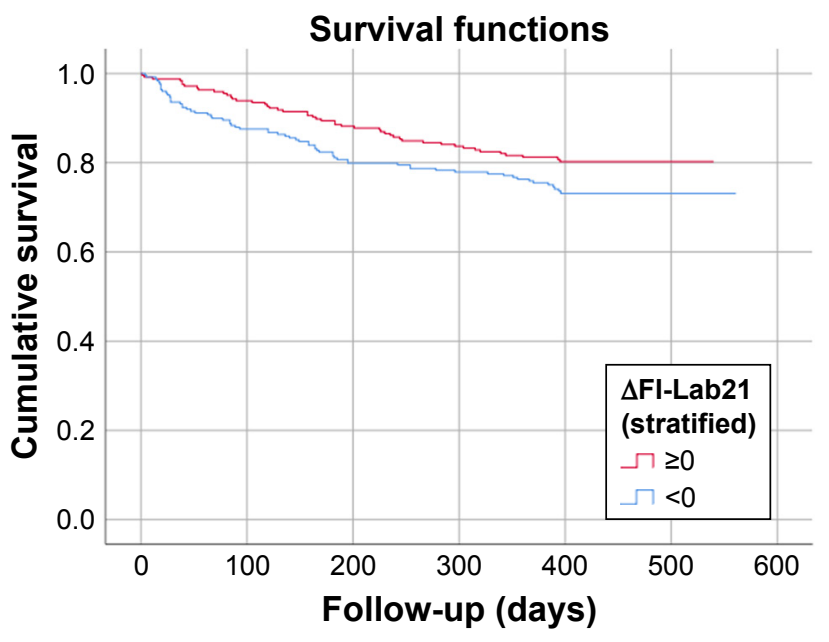

Figure 5 Kaplan-Meier survival function: change of the FI-Lab during the hospital stay $(\Delta \mathrm{FI}-\mathrm{Lab} 2 \mathrm{I})$ stratified into patients who suffered from an increase of their $\mathrm{Fl}$-Lab $(\Delta \mathrm{FI}$-Lab2 I $\geq 0)$ and those patients whose FI-Lab did not change or decreased $(\Delta \mathrm{FI}-\mathrm{Lab} 2 \mathrm{I}<0)$ during the hospital stay.

Abbreviation: FI-Lab2 I, $2 \mathrm{I}$-item frailty index based on laboratory blood and urine tests. hospital stay at the hospital geriatric wards. This indicates that not only the FI-Lab21 irrespective of whether assessed at admission to hospital or before discharge from hospital but also the change of the FI-Lab21 reveals predictive power for 6-month and 1-year mortality in hospitalized geriatric patients. Our data thus show that patients who did not suffer from a deterioration of their frailty severity, as assessed by the FI-Lab21, during their hospital stay, revealed a better survival compared with those patients whose frailty status, as assessed by the FI-Lab21, worsened during the hospital stay at the geriatric wards. Moreover, after adjustment of the analysis for age, sex, and FI-Lab2 $1_{\text {admission, }}$, we found an association of the quantity of improvement of the FI-Lab21 during the hospital stay and the reduction in 6-month and 1 -year mortality risk of the hospitalized geriatric patients. This indicates that also the changes of the FI-Lab21 during the hospital stay might be an interesting and additional tool to stratify mortality risk in hospitalized geriatric patients.

This study has some major strengths. To the best of our knowledge, this is the first study evaluating an FI based solely on laboratory parameters at different points in time for patients during hospital stay. The FI-Lab21 used in the present study was based solely on few routine laboratory parameters enabling easy implementation of this FI-Lab into clinical practice. Of note, the FI-Lab21 as evaluated in this study shows key characteristics of FI such as an upper limit of FI of $\sim 0.7 .^{43,44}$

This study has limitations. The study was a single-center analysis. Single-center studies might not be generalizable to a broad population. Of note, in some cases, single-center studies have been contradicted in subsequently larger multicenter studies. ${ }^{45}$ In addition, the predominant part of our cohort was Caucasian. Further investigation is needed to extrapolate our findings to other ethnic groups. Apparently, according to the findings of Chen et al, comorbidity burden, mobility-related disability, and the impact of acute and chronic diseases might have driven the FI-Lab21 ${ }_{\text {admission}}$, the FI-Lab2 $1_{\text {discharge }}$, and the change of the FI-Lab21 during the hospital stay, intensifying their predictive power on individuals risk at death. ${ }^{46}$ Furthermore, a total number of 21 health deficits as operationalized in our study might underestimate adverse outcomes. More robust estimates are measured with FIs consisting of 50 or more deficits. ${ }^{47}$ Thus, our data have to be considered with caution.

In conclusion, the FI-Lab21 irrespective of whether assessed at admission to hospital or before discharge from hospital and the changes of the FI-Lab21 during the hospital stay at the geriatric wards emerged as interesting and feasible 
Table 6 HR and HR adjusted for age, sex, and Fl-Lab2 $\mathrm{I}_{\mathrm{ad}}$ for each $0.0 \mathrm{I}$ increment in score of $\Delta \mathrm{FI}-\mathrm{Lab} 2 \mathrm{I}$ in relation to 6-month and I-year mortality

\begin{tabular}{|l|l|l|l|l|}
\hline$\Delta$ FI-Lab2I and mortality & HR $(\mathbf{9 5} \% \mathbf{C I})$ & P-value & Adjusted HR (95\% CI) & P-value \\
\hline Six-month mortality & $0.980(0.96 \mathrm{I}-0.999)$ & 0.040 & $0.94 \mathrm{I}(0.92 \mathrm{I}-0.96 \mathrm{I})$ & $<0.00 \mathrm{I}$ \\
\hline$\Delta \mathrm{FI}-$ Lab2I (per each 0.0I increment in score) & $0.987(0.970-\mathrm{I} .004)$ & 0.126 & $0.947(0.93 \mathrm{I}-0.965)$ & $<0.00 \mathrm{I}$ \\
\hline One-year mortality
\end{tabular}

Note: $\Delta \mathrm{FI}$-Lab2 I = FI-Lab2 $\mathrm{I}_{\text {admission }}-\mathrm{FI}$-Lab2 $\mathrm{I}_{\text {discharge }}$.

Abbreviations: Adjusted HR, hazard ratio adjusted for age, sex, and FI-Lab2I change of Fl-Lab during hospital stay.

approaches to stratify the risk for 6-month and 1-year mortality in hospitalized individuals on geriatric wards. Thereby, the FI-Lab21 evaluated at the point in time before discharge from hospital might be more powerful than the FI-Lab21 operationalized from the data obtained at admission to hospital in predicting 6-month and 1-year mortality in aforementioned group of persons. Furthermore, also the quantity of change of the FI-Lab21 during the hospital stay reveals predictive power for mortality in hospitalized geriatric patients.

\section{Acknowledgments}

We acknowledge the support by Deutsche Forschungsgemeinschaft and Friedrich-Alexander-Universität ErlangenNürnberg (FAU) within the funding program Open Access Publishing. The present work was performed in (partial) fulfillment of the requirements for obtaining the degree "Dr. med." by the Friedrich-Alexander-Universität ErlangenNürnberg (JJ).

\section{Disclosure}

The authors report no conflicts of interest in this work.

\section{References}

1. Rockwood K. A global clinical measure of fitness and frailty in elderly people. Can Med Assoc J. 2005;173(5):489-495.

2. Clegg A, Young J, Iliffe S, Rikkert MO, Rockwood K. Frailty in elderly people. Lancet. 2013;381(9868):752-762.

3. Evans SJ, Sayers M, Mitnitski A, Rockwood K. The risk of adverse outcomes in hospitalized older patients in relation to a frailty index based on a comprehensive geriatric assessment. Age Ageing. 2014;43(1): 127-132.

4. Mousa A, Savva GM, Mitnitski A, et al. Is frailty a stable predictor of mortality across time? Evidence from the cognitive function and ageing studies. Age Ageing. 2018;47(5):721-727.

5. Pilotto A, Rengo F, Marchionni N, et al. Comparing the prognostic accuracy for all-cause mortality of frailty instruments: a multicentre 1-year follow-up in hospitalized older patients. PLoS One. 2012;7(1):e29090.

6. Ritt M, Bollheimer LC, Sieber CC, Gaßmann KG. Prediction of one-year mortality by five different frailty instruments: a comparative study in hospitalized geriatric patients. Arch Gerontol Geriatr. 2016;66:66-72.

7. Theou O, Brothers TD, Mitnitski A, Rockwood K. Operationalization of frailty using eight commonly used scales and comparison of their ability to predict all-cause mortality. J Am Geriatr Soc. 2013;61(9):1537-1551.
8. Hoogendijk EO, Theou O, Rockwood K, Onwuteaka-Philipsen BD, Deeg DJH, Huisman M. Development and validation of a frailty index in the longitudinal aging study Amsterdam. Aging Clin Exp Res. 2017; 29(5):927-933.

9. Cesari M, Landi F, Vellas B, Bernabei R, Marzetti E. Sarcopenia and physical frailty: two sides of the same coin. Front Aging Neurosci. 2014;6:192.

10. Kemmler W, Teschler M, Goisser S, et al. Prevalence of sarcopenia in Germany and the corresponding effect of osteoarthritis in females 70 years and older living in the community: results of the FORMoSA study. Clin Interv Aging. 2015;10:1565-1573.

11. Dorner TE, Luger E, Tschinderle J, et al. Association between nutritional status (MNA ${ }^{\mathbb{B}}$-SF) and frailty (SHARE-FI) in acute hospitalised elderly patients. J Nutr Health Aging. 2014;18(3):264-269.

12. Christner S, Ritt M, Volkert D, Wirth R, Sieber CC, Gaßmann KG. Evaluation of the nutritional status of older hospitalised geriatric patients: a comparative analysis of a Mini Nutritional Assessment (MNA) version and the Nutritional Risk Screening (NRS 2002). J Hum Nutr Diet. 2016;29(6):704-713.

13. Hanlon P, Nicholl BI, Jani BD, Lee D, Mcqueenie R, Mair FS. Frailty and pre-frailty in middle-aged and older adults and its association with multimorbidity and mortality: a prospective analysis of 493737 UK Biobank participants. Lancet Public Health. 2018;3(7):e323-e332.

14. Halil M, Cemal Kizilarslanoglu M, Emin Kuyumcu M, Yesil Y, Cruz Jentoft AJ. Cognitive aspects of frailty: mechanisms behind the link between frailty and cognitive impairment. J Nutr Health Aging. 2015; 19(3):276-283.

15. Ní Mhaoláin AM, Fan CW, Romero-Ortuno R, et al. Frailty, depression, and anxiety in later life. Int Psychogeriatr. 2012;24(8):1265-1274.

16. Ritt M, Schülein S, Lubrich H, Bollheimer LC, Sieber CC, Gaßmann KG. High-technology based gait assessment in frail people: associations between spatio-temporal and three-dimensional gait characteristics with frailty status across four different frailty measures. J Nutr Health Aging. 2017;21(3):346-353.

17. Weiss CO. Frailty and chronic diseases in older adults. Clin Geriatr Med. 2011;27(1):39-52.

18. Morley JE, Vellas B, van Kan GA, et al. Frailty consensus: a call to action. J Am Med Dir Assoc. 2013;14(6):392-397.

19. Muscedere J, Andrew MK, Bagshaw SM, et al. Screening for frailty in Canada's health care system: a time for action. Can J Aging. 2016;35(03): 281-297.

20. Ritt M, Rádi KH, Schwarz C, Bollheimer LC, Sieber CC, Gaßmann KG. A comparison of frailty indexes based on a comprehensive geriatric assessment for the prediction of adverse outcomes. J Nutr Health Aging. 2016;20(7):760-767.

21. Ritt M, Schwarz C, Kronawitter V, et al. Analysis of Rockwood et al's clinical frailty scale and Fried et al's frailty phenotype as predictors of mortality and other clinical outcomes in older patients who were admitted to a geriatric ward. J Nutr Health Aging. 2015;19(10): 1043-1048.

22. Fried LP, Tangen CM, Walston J, et al. Frailty in older adults: evidence for a phenotype. J Gerontol A Biol Sci Med Sci.2001;56(3):M146-M157. 
23. Mitnitski AB, Mogilner AJ, Rockwood K. Accumulation of deficits as a proxy measure of aging. ScientificWorldJournal. 2001;1:323-336.

24. Rockwood K, Theou O, Mitnitski A. What are frailty instruments for? Age Ageing. 2015;44(4):545-547.

25. Ritt M, Ritt JI, Sieber CC, Gaßmann KG. Comparing the predictive accuracy of frailty, comorbidity, and disability for mortality: a 1-year followup in patients hospitalized in geriatric wards. Clin Interv Aging. 2017; 12:293-304.

26. Rockwood K, Andrew M, Mitnitski A. A comparison of two approaches to measuring frailty in elderly people. J Gerontol A Biol Sci Med Sci. 2007;62(7):738-743.

27. Rockwood K, Rockwood MR, Mitnitski A. Physiological redundancy in older adults in relation to the change with age in the slope of a frailty index. J Am Geriatr Soc. 2010;58(2):318-323.

28. Ritt M, Jäger J, Ritt J, Sieber CC, Gaßmann KG. Operationalizing a frailty index using routine blood and urine tests. Clin Interv Aging. 2017;12:1029-1040.

29. Blodgett JM, Theou O, Howlett SE, Rockwood K. A frailty index from common clinical and laboratory tests predicts increased risk of death across the life course. GeroScience. 2017;39(4):447-455.

30. Blodgett JM, Theou O, Howlett SE, Wu FC, Rockwood K. A frailty index based on laboratory deficits in community-dwelling men predicted their risk of adverse health outcomes. Age Ageing. 2016;45(4):463-468.

31. Howlett SE, Stanley J, Wong H, Mitnitski A, Rockwood K. A procedure to create a frailty index based on routinely-collected laboratory and clinical safety data in the setting of an Alzheimer's disease clinical trial. Alzheimers Dement. 2017;13(7):P1519.

32. Rockwood K, McMillan M, Mitnitski A, Howlett SE. A frailty index based on common laboratory tests in comparison with a clinical frailty index for older adults in long-term care facilities. J Am Med Dir Assoc. 2015; 16(10):842-847.

33. Klausen HH, Petersen J, Bandholm T, et al. Association between routine laboratory tests and long-term mortality among acutely admitted older medical patients: a cohort study. BMC Geriatr. 2017;17(1):62.

34. Hubbard RE, Peel NM, Samanta M, Gray LC, Mitnitski A, Rockwood K. Frailty status at admission to hospital predicts multiple adverse outcomes. Age Ageing. 2017;46(5):801-806.
35. Swets JA. Measuring the accuracy of diagnostic systems. Science. 1988; 240(4857):1285-1293.

36. Hanley JA, McNeil BJ. A method of comparing the areas under receiver operating characteristic curves derived from the same cases. Radiology. 1983;148(3):839-843.

37. Basile G, Catalano A, Mandraffino G, et al. Frailty modifications and prognostic impact in older patients admitted in acute care. Aging Clin Exp Res. Epub 2018 June 26.

38. Fairhall N, Sherrington C, Kurrle SE, Lord SR, Lockwood K, Cameron ID. Effect of a multifactorial interdisciplinary intervention on mobility-related disability in frail older people: randomised controlled trial. BMC Med. 2012;10(1):120.

39. Fiatarone MA, O'Neill EF, Ryan ND, et al. Exercise training and nutritional supplementation for physical frailty in very elderly people. N Engl J Med. 1994;330(25):1769-1775.

40. Kim H. Interventions for frailty and sarcopenia in community-dwelling elderly women. Nihon Ronen Igakkai Zasshi. 2012;49(6):726-730.

41. Ng TP, Feng L, Nyunt MS, et al. Nutritional, physical, cognitive, and combination interventions and frailty reversal among older adults: a randomized controlled trial. Am J Med. 2015;128(11):1225-1236.

42. Howlett SE, Rockwood MR, Mitnitski A, Rockwood K. Standard laboratory tests to identify older adults at increased risk of death. BMC Med. 2014;12(1):171.

43. Armstrong JJ, Mitnitski A, Launer LJ, White LR, Rockwood K. Frailty in the Honolulu-Asia aging study: deficit accumulation in a male cohort followed to $90 \%$ mortality. J Gerontol A Biol Sci Med Sci. 2015;70(1): 125-131.

44. Searle SD, Mitnitski A, Gahbauer EA, Gill TM, Rockwood K. A standard procedure for creating a frailty index. BMC Geriatr. 2008; $8(1): 24$.

45. Bellomo R, Warrillow SJ, Reade MC. Why we should be wary of single-center trials. Crit Care Med. 2009;37(12):3114-3119.

46. Chen C, Sia I, Ma HM, et al. The synergistic effect of functional status and comorbidity burden on mortality: a 16-year survival analysis. PLoS One. 2014;9(8):e106248.

47. Rockwood K, Mitnitski A. How might deficit accumulation give rise to frailty? J Frailty Aging. 2012;1(1):8-12.
Clinical Interventions in Aging

\section{Publish your work in this journal}

Clinical Interventions in Aging is an international, peer-reviewed journal focusing on evidence-based reports on the value or lack thereof of treatments intended to prevent or delay the onset of maladaptive correlates of aging in human beings. This journal is indexed on PubMed Central, MedLine,

\section{Dovepress}

CAS, Scopus and the Elsevier Bibliographic databases. The manuscript management system is completely online and includes a very quick and fair peer-review system, which is all easy to use. Visit http://www.dovepress. com/testimonials.php to read real quotes from published authors. 